Draft Version November 10, 2018

Preprint typeset using $\mathrm{LAT}_{\mathrm{EX}}$ style emulateapj v. 14/09/00

\title{
VERTICAL STRUCTURE OF STATIONARY ACCRETION DISKS WITH A LARGE-SCALE MAGNETIC FIELD
}

\author{
G.S. Bisnovatyi-Kogan ${ }^{1} \&$ R.V.E. Lovelace ${ }^{2}$ \\ Draft version November 10, 2018
}

\begin{abstract}
In earlier works we pointed out that the disk's surface layers are non-turbulent and thus highly conducting (or non-diffusive) because the hydrodynamic and/or magnetorotational (MRI) instabilities are suppressed high in the disk where the magnetic and radiation pressures are larger than the plasma thermal pressure. Here, we calculate the vertical profiles of the stationary accretion flows (with radial and azimuthal components), and the profiles of the large-scale, magnetic field taking into account the turbulent viscosity and diffusivity and the fact that the turbulence vanishes at the surface of the disk. Also, here we require that the radial accretion speed be zero at the disk's surface and we assume that the ratio of the turbulent viscosity to the turbulent magnetic diffusivity is of order unity. Thus at the disk's surface there are three boundary conditions. As a result, for a fixed dimensionless viscosity $\alpha$-value, we find that there is a definite relation between the ratio $\mathcal{R}$ of the accretion power going into magnetic disk winds to the viscous power dissipation and the midplane plasma- $\beta$, which is the ratio of the plasma to magnetic pressure in the disk.

For a specific disk model with $\mathcal{R}$ of order unity we find that the critical value required for a stationary solution is $\beta_{c} \approx 2.4 r /(\alpha h)$, where $h$ the disk's half thickness. For weaker magnetic fields, $\beta>\beta_{c}$, we argue that the poloidal field will advect outward while for $\beta<\beta_{c}$ it will advect inward. Alternatively, if the disk wind is negligible $(\mathcal{R} \ll 1)$, there are stationary solutions with $\beta \gg \beta_{c}$.
\end{abstract}

Subject headings: accretion, accretion disks — galaxies: jets — magnetic fields - MHD - X-rays: binaries

\section{INTRODUCTION}

Analysis of the diffusion and advection of a large-scale magnetic field in a accretion disk with a turbulent viscosity and magnetic diffusivity arising from the magnetorotational instability (MRI) shows that a weak large-scale field diffuses outward rapidly (van Ballegooijen 1989; Lubow, Papaloizou, \& Pringle 1994). We mention but do not consider here the opposite limit where the magnetic field is sufficiently strong that it suppresses the MRI instability so that the disk is non-turbulent but accretion occurs due to angular momentum outflow to a magnetic disk wind or jet (Lovelace, Romanova, \& Newman 1994). Earlier, Bisnovatyi-Kogan and Lovelace (2007) pointed out that the disk's surface layers are highly conducting because the MRI instability is suppressed in this region where the magnetic and radiative energy-densities are larger than the thermal gas energy-density. Rothstein and Lovelace (2008) analyzed this problem in further detail and discussed the connections with global and shearing box magnetohydrodynamic (MHD) simulations of the MRI. Lovelace, Rothstein, \& Bisnovatyi-Kogan (2009; hereafter LRBK) developed an analytic model for the vertical $(z)$ profiles of the stationary accretion flows (with radial and azimuthal components), and the profiles of the large-scale, magnetic field taking into account the turbulent viscosity and diffusivity due to the MRI and the fact that the turbulence vanishes at the surface of the disk.

Here, we require that the radial accretion speed be zero at the disk's surface, and we assume that the ratio of the turbulent viscosity to the turbulent magnetic diffusivity is of order unity as suggested by MHD shearing-box simula- tions (Guan \& Gammie 2009). For a fixed dimensionless viscosity $\alpha$-value, we find that there is a definite relation between the ratio $\mathcal{R}$ of the accretion power going into magnetic disk winds to the viscous power dissipation and the midplane plasma- $\beta$, which is the ratio of the plasma to magnetic pressure in the disk.

Section 2 discusses the model for the flow and ordered magnetic field in a viscous diffusive disk. Section 3 discusses the solutions for a specific disk model. Section 4 gives the conclusions.

\section{THEORY}

Following LRBK we consider the non-ideal magnetohydrodynamics of a thin axisymmetric, viscous, resistive disk threaded by a large-scale dipole-symmetry magnetic field B. We use a cylindrical $(r, \phi, z)$ inertial coordinate system in which the time-averaged magnetic field is $\mathbf{B}=B_{r} \hat{\mathbf{r}}+B_{\phi} \hat{\boldsymbol{\phi}}+B_{z} \hat{\mathbf{z}}$, and the time-averaged flow velocity is $\mathbf{v}=v_{r} \hat{\mathbf{r}}+v_{\phi} \hat{\boldsymbol{\phi}}+v_{z} \hat{\mathbf{z}}$. The main equations are

$$
\begin{aligned}
\rho \frac{d \mathbf{v}}{d t} & =-\nabla p+\rho \mathbf{g}+\frac{1}{c} \mathbf{J} \times \mathbf{B}+\mathbf{F}^{\nu}, \\
\frac{\partial \mathbf{B}}{\partial t} & =\nabla \times(\mathbf{v} \times \mathbf{B})-\nabla \times(\eta \nabla \times \mathbf{B}) .
\end{aligned}
$$

These equations are supplemented by the continuity equation, $\nabla \cdot(\rho \mathbf{v})=0$, by $\nabla \times \mathbf{B}=4 \pi \mathbf{J} / c$, and by $\nabla \cdot \mathbf{B}=0$. Here, $\eta$ is the magnetic diffusivity, $\mathbf{F}^{\nu}=-\nabla \cdot T^{\nu}$ is the viscous force with $T_{j k}^{\nu}=-\rho \nu\left(\partial v_{j} / \partial x_{k}+\partial v_{k} / \partial x_{j}-(2 / 3) \delta_{j k} \nabla\right.$. v) (in Cartesian coordinates), and $\nu$ is the kinematic viscosity. For simplicity, in place of an energy equation we consider the adiabatic dependence $p \propto \rho^{\gamma}$, with $\gamma$ the adiabatic index.

\footnotetext{
${ }^{1}$ Space Research Institute, Russian Academy of Sciences, Moscow, Russia; gkogan@mx.iki.rssi.ru
}

2 Department of Astronomy, Cornell University, Ithaca, NY 14853-6801; RVL1@cornell.edu 
We assume that both the viscosity and the diffusivity are due to magneto-rotational (MRI) turbulence in the disk so that

$$
\nu=\mathcal{P} \eta=\alpha \frac{c_{s 0}^{2}}{\Omega_{K}} g(z),
$$

where $\mathcal{P}$ is the magnetic Prandtl number of the turbulence assumed a constant of order unity (Bisnovatyi-Kogan \& Ruzmaikin 1976), $\alpha \leq 1$ is the dimensionless ShakuraSunyaev (1973) parameter, $c_{s 0}$ is the midplane isothermal sound speed, $\Omega_{K} \equiv\left(G M / r^{3}\right)^{1 / 2}$ is the Keplerian angular velocity of the disk, and $M$ is the mass of the central object. The function $g(z)$ accounts for the absence of turbulence in the surface layer of the disk (Bisnovatyi-Kogan \& Lovelace 2007; Rothstein \& Lovelace 2008). In the body of the disk $g=1$, whereas at the surface of the disk, at say $z_{S}, g$ tends over a short distance to a very small value $\sim 10^{-8}$, effectively zero, which is the ratio of the Spitzer diffusivity of the disk's surface layer to the turbulent diffusivity of the body of the disk. At the disk's surface the density is much smaller than its midplane value.

We consider stationary solutions of equations (1) and (2) for a weak large-scale magnetic field. These can be greatly simplified for thin disks where the disk half-thickness, of the order of $h \equiv c_{s 0} / \Omega_{K}$, is much less than $r$. Thus we have the small parameter

$$
\varepsilon=\frac{h}{r}=\frac{c_{s 0}}{v_{K}} \ll 1 .
$$

It is useful in the following to use the dimensionless height $\zeta \equiv z / h$. The midplane plasma beta is taken to be

$$
\beta \equiv \frac{4 \pi \rho_{0} c_{s 0}^{2}}{B_{0}^{2}}
$$

where $\beta=c_{s 0}^{2} / v_{A 0}^{2}, v_{A 0}=B_{0} /\left(4 \pi \rho_{0}\right)^{1 / 2}$ is the midplane Alfvén velocity. Note that the conventional definition of beta is $2 \beta$. The rough condition for the MRI instability and the associated turbulence in the disk is $\beta \gtrsim 1$ (Balbus \& Hawley 1998) and this is assumed here.

The three magnetic field components are assumed to be of comparable magnitude on the disk's surface, but $B_{r}=0=B_{\phi}$ on the midplane. On the other hand the axial magnetic field changes by only a small almount going from the midplane to the surface, $\Delta B_{z} \sim \varepsilon B_{r} \ll B_{z}$ (from $\nabla \cdot \mathbf{B}=0$ ) so that $B_{z} \approx$ const inside the disk. As a consequence, the $\partial B_{j} / \partial r$ terms in the magnetic force in equation (1) can all be dropped in favor of the $\partial B_{j} / \partial z$ terms (with $j=r, \phi$ ). It is important to keep in mind that $B_{j}$ is the large scale field; the approximation does not apply to the small-scale field which gives the viscosity and diffusivity. The three velocity components are assumed to satisfy $v_{z}^{2} \ll c_{s 0}^{2}$ and $v_{r}^{2} \ll v_{\phi}^{2}$. Consequently, $v_{\phi}(r, z)$ is close in value to the Keplerian value $v_{K}(r) \equiv(G M / r)^{1 / 2}$, except in the outer disk layers where the radial magnetic force may be comparable with the centrifugal and gravitational forces. We normalize the field components by $B_{0}=B_{z}(r, z=0)$, with $b_{r}=B_{r} / B_{0}, b_{\phi}=B_{\phi} / B_{0}$, and $b_{z}=B_{z} / B_{0} \approx 1$. Also, we define $u_{\phi} \equiv v_{\phi}(r, z) / v_{K}(r)$ and the accretion speed $u_{r} \equiv-v_{r} /\left(\alpha c_{s 0}\right)$. For the assumed dipole field symmetry, $b_{r}$ and $b_{\phi}$ are odd functions of $\zeta$ whereas $u_{r}$ and $u_{\phi}$ are even functions.

Integration over the vertical extent of the disk gives the average accretion speed

$$
\bar{u}_{r}=u_{0}-\frac{2 b_{\phi S+}}{\alpha \beta \tilde{\Sigma}},
$$

(LRBK) which is the sum of a viscous contribution, $u_{0} \equiv$ $3 \varepsilon k_{\nu}$ (with $k_{\nu}$ a numerical constant of order unity), and a magnetic contribution $\left(\propto b_{\phi S+}\right)$ due to the loss of angular momentum from the surface of the disk where necessarily $b_{\phi S+} \leq 0$ (Lovelace, Romanova, \& Newman 1994).

We assume $p \propto \rho^{\gamma}$ so that the vertical hydrostatic equilibrium gives

$$
\tilde{\rho}=\frac{\rho}{\rho_{0}}=\left(1-\frac{(\gamma-1) \zeta^{2}}{2 \gamma}\right)^{1 /(\gamma-1)},
$$

for $\beta \gg 1$. The density goes to zero at $\zeta_{m}=[2 \gamma /(\gamma-$ $1)]^{1 / 2}$. However, before this distance is reached the MRI turbulence is suppressed, and $g(\zeta)$ in equation (3) is effectively zero.

The different components of equations (1) and (2) can be combined (LRBK) to give the following equation for the radial accretion speed,

$$
\begin{array}{r}
\alpha^{4} \beta^{2} \frac{\partial^{2}}{\partial \zeta^{2}}\left(g \frac{\partial}{\partial \zeta}\left(\tilde{\rho} g \frac{\partial}{\partial \zeta}\left(\frac{1}{\tilde{\rho}} \frac{\partial}{\partial \zeta}\left(\tilde{\rho} g \frac{\partial u_{r}}{\partial \zeta}\right)\right)\right)\right) \\
-\alpha^{2} \beta \mathcal{P} \frac{\partial^{2}}{\partial \zeta^{2}}\left(g \frac{\partial}{\partial \zeta}\left(\tilde{\rho} g \frac{\partial}{\partial \zeta}\left(\frac{u_{r}}{\tilde{\rho} g}\right)\right)\right) \\
-\alpha^{2} \beta \mathcal{P} \frac{\partial^{2}}{\partial \zeta^{2}}\left(\frac{1}{\tilde{\rho}} \frac{\partial}{\partial \zeta}\left(\tilde{\rho} g \frac{\partial u_{r}}{\partial \zeta}\right)\right) \\
+\alpha^{2} \beta^{2} \frac{\partial^{2}}{\partial \zeta^{2}}\left(\tilde{\rho} g\left(u_{r}-g u_{0}\right)\right)+\mathcal{P}^{2} \frac{\partial^{2}}{\partial \zeta^{2}}\left(\frac{u_{r}}{\tilde{\rho} g}\right) \\
+3 \beta \mathcal{P}^{2} \frac{u_{r}}{g}=0 .
\end{array}
$$

The equation can be integrated from $\zeta=0$ out to the surface of the disk $\zeta_{S}$ where boundary conditions apply.

For specificity we take $g(\zeta)=\left(1-\zeta^{2} / \zeta_{S}^{2}\right)^{\delta}$, where $\zeta_{S}<\zeta_{m}$ and $\delta$ is a constant. That is, we neglect the ratio of the Spitzer diffusivity on the surface of the disk to its value in the central part of the disk. An estimate of $\zeta_{S}$ can be made by noting that $\beta(\zeta)=4 \pi p(\zeta) / B_{0}^{2}=\beta(\tilde{\rho})^{\gamma} \approx 1$ at $\zeta_{S}$. This gives $\zeta_{S}^{2} / \zeta_{m}^{2}=1-\beta^{-(\gamma-1) / \gamma}$ and $\rho_{S} / \rho_{0}=\beta^{-1 / \gamma}$.

\subsection{Boundary Conditions}

We consider only the solutions which have net mass accretion, $\dot{M}=4 \pi r h \rho_{0} \alpha c_{s 0} \tilde{\Sigma} \bar{u}_{r}>0$, and have $b_{\phi} \leq 0$ on the disk's surface. This condition on $b_{\phi S+}$ corresponds to an efflux of angular momentum and energy (or their absence) from the disk to its corona rather than the reverse. The condition $b_{\phi S+} \leq 0$ is the same as $\bar{u}_{r} \geq u_{0}$, where $u_{0}$ is the minimum (viscous) accretion speed. Note that $\mathcal{R}=\bar{u}_{r} / u_{0}-1$ is the ratio of the accretion power which goes into the disk winds to the viscous power dissipation. Clearly, the condition on $b_{\phi S+}$ implies that $\dot{M}>0$ so that there is only one condition. In general there is a continuum of values of $b_{\phi S} \leq 0$ for the considered solutions inside the disk. The value of $b_{\phi S}$ can be determined by matching the calculated fields $b_{r S}$ and $b_{\phi S}$ onto an external field and flow as discussed in LRBK and here in $\S 3$.

LRBK showed that there is no jump in $b_{r}$ across the conducting surface layer. This implies that $\partial u_{r} /\left.\partial \zeta\right|_{\zeta_{S}}=0$, which represents a first boundary condition of the solution of equation (8). The equations inter-reltaing $b_{r}, b_{\phi}$, $u_{r}$, and $\delta u_{\phi}$ are summarized in the Appendix. A second boundary condition, $\left.u_{r}\right|_{\zeta_{S}}=0$, follows from equation 12 
of LRBK evaluated just outside of the conducting layer. A third boundary condition, $\partial b_{\phi} /\left.\partial \zeta\right|_{\zeta_{S-}}=0$, is derived in LRBK.

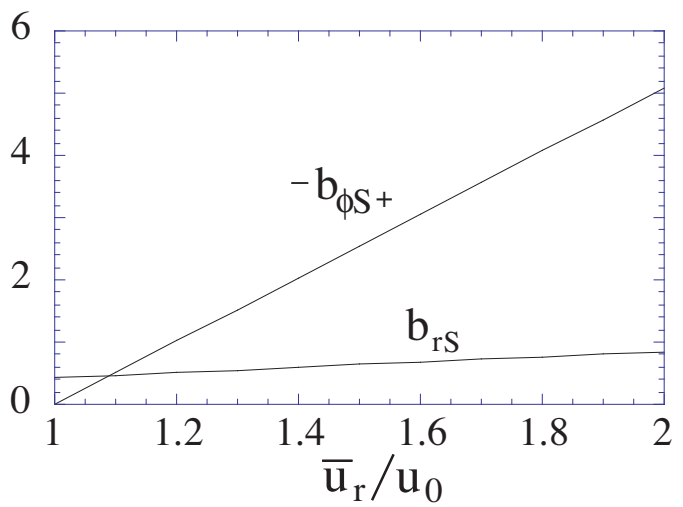

Fig. 1.- Radial and toroidal field components normalized to $B_{z}(r, 0)$ at the disk's surface as a function of the average accretion speed $\bar{u}_{r}$ (normalized by the viscous accretion speed $u_{0}$ ). For this plot $\beta=240$ and $\mathcal{P}=1$.

\section{SPECIFIC SOLUTIONS}

Here, to simplify the analysis we consider the limit where $\gamma \rightarrow \infty$ in equation (7) and $\delta \rightarrow 0$ in equation (18) of LRBK. Then, $\zeta_{S} \rightarrow \zeta_{m}$ and both $\tilde{\rho}$ and $g$ are unit step functions going to zero at $\zeta_{m}=\sqrt{2}$. Also, $\bar{u}_{r}=\left\langle u_{r}\right\rangle$ and $\tilde{\Sigma}=\sqrt{2}$. Thus the above physical condition $\bar{u}_{r} \geq 3 \varepsilon k_{\nu}=u_{0}$ implies that $b_{r S} \geq u_{0} \zeta_{S} \mathcal{P}$ from equation (13) of LRBK. We assume $k_{p} \equiv-\partial \ln p / \partial \ln r$ and $k_{\nu}=1$.

The solutions to equation (8) are $u_{r} \propto \exp \left(i k_{j} \zeta\right.$ ) (with $j=1,2,3)$, where

$$
\alpha^{4} \beta^{2}\left(k_{j}^{2}\right)^{3}+2 \mathcal{P} \alpha^{2} \beta\left(k_{j}^{2}\right)^{2}+\left(\alpha^{2} \beta^{2}+\mathcal{P}^{2}\right) k_{j}^{2}-3 \beta \mathcal{P}^{2}=0,
$$

is a cubic in $k_{j}^{2}$. The discriminant of the cubic is negative so that there is one real root, $k_{1}^{2}$, and a complex conjugate pair of roots, $k_{2,3}^{2}$. Because $u_{r}$ is an even function of $\zeta$ we can write

$$
\begin{aligned}
u_{r} & =a_{1} \cos \left(k_{0} \zeta\right)+a_{2} \cos \left(k_{r} \zeta\right) \cosh \left(k_{i} \zeta\right) \\
& +a_{3} \sin \left(k_{r} \zeta\right) \sinh \left(k_{i} \zeta\right)
\end{aligned}
$$

where $k_{0}=\sqrt{k_{1}^{2}}, k_{r}=\operatorname{Re}\left(\sqrt{k_{2}^{2}}\right)$, and $k_{i}=\operatorname{Im}\left(\sqrt{k_{2}^{2}}\right)$.

We consider a thin disk, $\varepsilon=h / r=0.05$, and a viscosity parameter $\alpha=0.1$. Figure 1 shows the dependences of the surface field components on the average accretions speed for $\beta=240$ and $\mathcal{P}=1$. The $b_{\phi S}$ dependence is given by equation (6) and is independent of $\mathcal{P}$ while the $b_{r S}$ is given by $b_{r S}=\mathcal{P} \zeta_{S} \bar{u}_{r}$ from LRBK (equation 13 ).

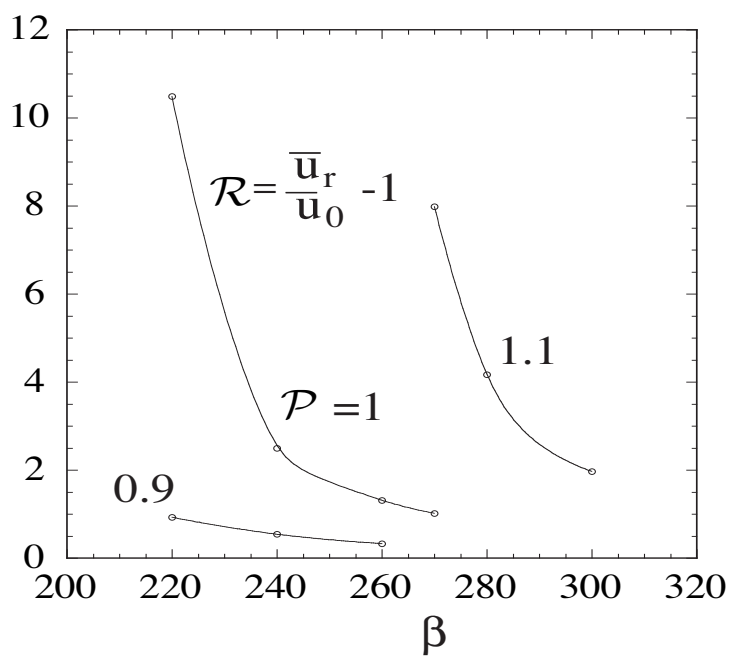

FIG. 2.- Summary of solutions to equation (8) which satisfy the boundary conditions of $\S 2.1 . \bar{u}_{r}$ is the average accretion speed and $u_{0}$ is the accretion speed in the absence of an ordered magnetic field. The curves are smooth lines connecting the marked points. The physically relevant solutions have $\mathcal{R} \lesssim \mathcal{O}\left[2 \sqrt{2} \pi /\left(\alpha \beta u_{0}\right)\right]$ which is about 2.5 for $\alpha=0.1, \beta=240$, and $u_{0}=0.15$.

There are three boundary conditions at the surface of the disk. Additionally, there is the ratio $\mathcal{R}$ of the accretion power going into magnetic disk winds to the viscous power dissipation. The solution for $u_{r}(\zeta)$ has three unknown coefficients, $a_{1}, a_{2}$. \& $a_{3}$ which are dependent on $\alpha, \mathcal{P}$, and $\beta$. If $\alpha$ and $\mathcal{P}$ are fixed, then there is a definite relation between $\mathcal{R}$ and $\beta$. Figure 2 shows this relation determined numerically.

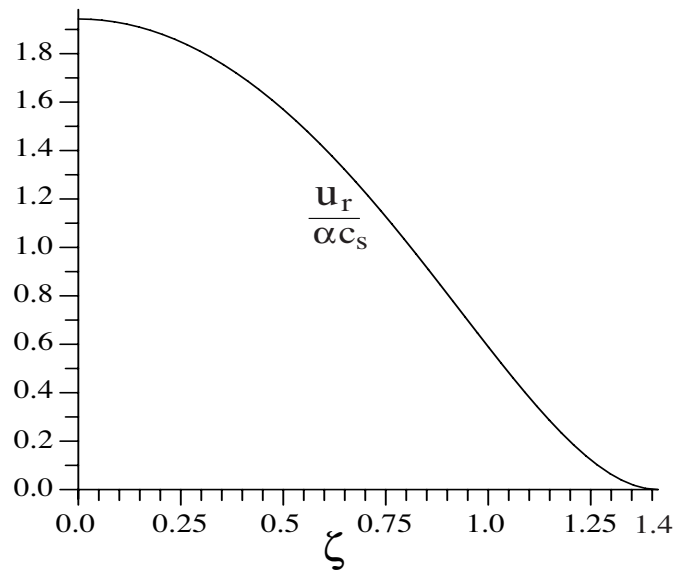

FIG. 3.- Radial inflow speed $u_{r}=-v_{r}$ (normalized to $\alpha c_{s 0}$ ) as a function of $\zeta=z / h$ for $\beta=240$ and $\mathcal{P}=1$. The surface of the disk is at $\zeta=\sqrt{2}$.

Figures 3 shows the vertical profile of the accretion flow for $\beta=240$. The profiles of $\delta u_{\phi}=\left(v_{\phi}-v_{K}\right) / v_{K}, b_{r}, b_{\phi}$ follow from the equations in the Appendix.

The value of $b_{\phi S+} \leq 0$ or $\bar{u}_{r} / u_{0}$ is not fixed by the solution for the field and flow inside the disk. Its value can be determined by matching the calculated surface fields $b_{r S}$ and $b_{\phi S+}$ onto an external magnetic wind or jet solution. Stability of the wind or jet solution to current driven kinking is predicted to limit the ratio of the toroidal to axial magnetic field components at the disk's surface $\left|b_{\phi S+}\right|$ to 
values $\lesssim \mathcal{O}(2 \pi)$ (Hsu \& Bellan 2002; Nakamura, Li, \& Li 2007). Recall that $\mathcal{R}=\bar{u}_{r} / u_{0}-1=2\left|b_{\phi S+}\right| /\left(\alpha \beta \sqrt{2} u_{0}\right)$ is the ratio of the accretion power going into the disk wind to the viscous dissipation in the disk. For the mentioned upper limit on $\left|b_{\phi S+}\right|, \mathcal{R} \lesssim \mathcal{O}\left[2 \sqrt{2} \pi /\left(\alpha \beta u_{0}\right)\right] \approx 2.5$. This implies a critical value of $\beta$ for a stationary disk solution (for $\mathcal{P}=1), \beta_{c} \approx 1.2(\alpha \varepsilon)^{-1}$. For $\alpha=0.1$ and $\varepsilon=0.05$, $\beta_{c} \approx 240$.

For $\beta \lessgtr \beta_{c}$, the disk and large-scale magnetic field is not be in a stationary state. Equation (6) for the average accretion speed still applies and can be written as $\bar{u}_{r}=u_{0}+u_{B}$ where $u_{B}$ is the magnetic contribution to the accretion due to the outflow of angular momentum from the disk's surfaces. In general, $u_{B}$ is an increasing function of the magnetic field strength if $\mathcal{R} \sim 1$ (Lovelace et al. 1994). For this reason for $\beta<\beta_{c}$ the ordered poloidal magnetic field threading the disk will be advected inward in the disk while for $\beta>\beta_{c}$ the field will advect outward. Thus, $\beta_{c}$ acts as a threshold value for the buildup of a large scale field in the inner regions of an accretion disk.

Another possibility is that the disk winds are negligible so that $\mathcal{R} \ll 1$. Figure 2 suggests that in this limit we have a stable stationary solutions with $\beta$ values much larger than the mentioned critical value.

\section{CONCLUSIONS}

For a specific disk model with turbulent Prandtl number $\mathcal{P}=1$ and $\mathcal{R} \sim 1$,we show that there is a critical value $\beta_{c} \approx 1.2 r /(\alpha h)$ for a stationary disk threaded by a largescale magnetic field. For $\alpha=0.1$ and $\varepsilon=0.05, \beta_{c} \approx 240$. For $\beta<\beta_{c}$, we argue that that the large-scale field will be advected inward and lead to the build up of the ordered field in the central region of the disk. For $\beta>\beta_{c}$, the ordered field will advect outward. Alternatively, if the disk wind is negligible so that $\mathcal{R} \ll 1$, stationary disk equilibria with a large scale field are possible for $\beta$ much larger than the mentioned $\beta_{c}$.

The 3D MHD simulations of Beckwith, Hawley, \& Krolik (2009) show the inward advection of a significant fraction of the initial unipolar vertical magnetic flux threading a MRI unstable plasma torus around a black hole. The initial average $\beta$ in the torus is 100 which is significantly less than the critical value estimated here. A broader range of simulations for much larger initial values of $\beta$ would be needed to determine if the initial flux diffuses outward. Axisymmetric MHD simulations of Romanova et al. (2011) show episodes of enhanced accretion on the disk surfaces for the case of an MRI unstable disk around a rotating magnetized stars. However, the presence of the stellar magnetic field complicates the analysis of flux transport in the disk.

We have shown that the presence of a large scale poloidal magnetic field in an accretion disk around a black hole not only plays a decisive role in the jet formation, particle acceleration, and observed hard energy radiation (BoschRamon 2011), but also creates the possibility for a new type of non-stationary behavior in these objects, which could be related to appearance of different spectral and luminosity states and transitions between them as observed in microquasars (Malzac and Belmont 2009).

\section{ACKNOWLEDGMENTS}

The work of G.S.B.-K. was partially supported by RFBR grants 08-02-00491 and 08-02-90106, RAN Program "Formation and evolution of stars and galaxies." R.V.E.L was supported in part by NASA grants NNX10AF63G and NNX11AF33G and by NSF grant AST-1008636.

\section{REFERENCES}

Balbus, S.A., \& Hawley, J.F. 1998, Rev. Mod. Phys., 70, 1

Beckwith, K., Hawley, J.F., \& Krolik, J.H. 2009, ApJ, 707, 428

Bisnovatyi-Kogan, G. S., \& Ruzmaikin, A. A. 1976, Ap\&SS, 42, 401

Bisnovatyi-Kogan, G.S., \& Lovelace, R.V.E. 2007, ApJ, 667, L167

Bosch-Ramon, V. 2011, arXiv1106.2059

Guan, X., \& Gammie, C.F. 2009, ApJ, 697, 1901

Hsu, S.C., \& Bellan, P.M. 2002, MNRAS, 334, 257

Lovelace, R.V.E., Berk, H.L., \& Contopoulos, J. 1991, ApJ, 379, 696

Lovelace, R.V.E., Romanova, M.M., \& Newman, W.I. 1994, ApJ, 437, 136 - 1997, ApJ, 484, 628

Lovelace, R.V.E., Rothstein, D.M., \& Bisnovatyi-Kogan, G.S. 2009, ApJ, 701, 885 (LRBK)
Lubow, S. H., Papaloizou, J. C. B., \& Pringle, J. E. 1994, MNRAS, 267,235

Malzac, J \& Belmont, R. 2009, MNRAS, 392, 570

Nakamura, M., Li, H., \& Li, S. 2007, ApJ, 656, 721

Pariev, V.I., Colgate, S.A., \& Finn, J.M. 2007, ApJ, 658, 129

Romanova, M.M., Ustyugova, G.V., Koldoba, A.V., \& Lovelace, R.V.E. 2011, MNRAS, in press

Rothstein, D.M., \& Lovelace, R.V.E. 2008, ApJ, 677, 1221

Shakura, N.I., \& Sunyaev, R.A. 1973, A\&A, 24, 337

van Ballegooijen, A. A. 1989, in Accretion Disks and Magnetic Fields in Astrophysics, ed. G. Belvedere ( Dordrecht: Kluwer), 99

\section{APPENDIX}

\section{SUBSIDIARY EQUATIONS}

Here, we summarize the relations between the field and velocity components. From the radial force balance,

$$
\frac{\partial b_{r}}{\partial \zeta}=\frac{\beta \tilde{\rho}}{\varepsilon}\left(1-k_{p} \varepsilon^{2}-u_{\phi}^{2}\right)+\alpha^{2} \beta \frac{\partial}{\partial \zeta}\left(\tilde{\rho} g \frac{\partial u_{r}}{\partial \zeta}\right),
$$

where $k_{p} \equiv-d \ln p / d \ln r$ is assumed positive and of order unity. The $\phi$-component of equation (2) gives

$$
\frac{\partial b_{\phi}}{\partial \zeta}=\frac{\alpha \beta \tilde{\rho}}{2}\left(3 \varepsilon k_{\nu} g-u_{r}\right)-\frac{\alpha \beta}{\varepsilon} \frac{\partial}{\partial \zeta}\left(\tilde{\rho} g \frac{\partial u_{\phi}}{\partial \zeta}\right),
$$

where $k_{\nu} \equiv d \ln \left(\rho c_{s 0}^{2} r^{2} / h\right) / d \ln (r)>0$ is also assumed to be of order unity.

The toroidal component of Ohm's law gives

$$
\frac{\partial b_{r}}{\partial \zeta}=\frac{\mathcal{P}}{g} u_{r} .
$$


The other components of Ohm's law give

$$
\frac{\partial u_{\phi}}{\partial \zeta}=\frac{3 \varepsilon}{2} b_{r}-\frac{\alpha \varepsilon}{\mathcal{P}} \frac{\partial}{\partial \zeta}\left(g \frac{\partial b_{\phi}}{\partial \zeta}\right) .
$$

Combining equations (A1) and (A3) gives

$$
u_{r}=\frac{\beta \tilde{\rho} g}{\varepsilon \mathcal{P}}\left(1-k_{p} \varepsilon^{2}-u_{\phi}^{2}\right)+\frac{\alpha^{2} \beta g}{\mathcal{P}} \frac{\partial}{\partial \zeta}\left(\tilde{\rho} g \frac{\partial u_{r}}{\partial \zeta}\right) .
$$

For thin disks, $\varepsilon \ll 1$, and $\beta>1$, we have $u_{\phi}=1+\delta u_{\phi}$ with $\left(\delta u_{\phi}\right)^{2} \ll 1$. Consequently,

$$
\delta u_{\phi}=-\frac{k_{p} \varepsilon^{2}}{2}-\frac{\varepsilon \mathcal{P} u_{r}}{2 \beta \tilde{\rho} g}+\frac{\alpha^{2} \varepsilon}{2 \tilde{\rho}} \frac{\partial}{\partial \zeta}\left(\tilde{\rho} g \frac{\partial u_{r}}{\partial \zeta}\right),
$$

to a good approximation. 